\title{
Measuring dark energy spatial inhomogeneity with supernova data
}

\author{
Asantha Cooray ${ }^{1}$, Daniel E. Holz ${ }^{2}$, and Robert Caldwell ${ }^{3}$ \\ ${ }^{1}$ Department of Physics and Astronomy, University of California, Irvine, CA 92697 \\ ${ }^{2}$ Theoretical Division, Los Alamos National Laboratory, Los Alamos, NM 87545 and \\ ${ }^{3}$ Department of Physics \& Astronomy, Dartmouth College, 6127 Wilder Laboratory, Hanover, NH 03755
}

(Dated: October 29, 2018)

\begin{abstract}
The gravitational lensing distortion of distant sources by the large-scale distribution of matter in the Universe has been extensively studied. In contrast, very little is known about the effects due to the large-scale distribution of dark energy. We discuss the use of Type Ia supernovae as probes of the spatial inhomogeneity and anisotropy of dark energy. We show that a shallow, almost all-sky survey can limit rms dark energy fluctuations at the horizon scale down to a fractional energy density of $\sim 10^{-4}$.
\end{abstract}

Introduction - Type Ia supernovae (SNe) are thought to be excellent standard candles, with a calibrated luminosity distance known to $\sim 15 \%$ [1]. By using these $\mathrm{SNe}$ to measure relative distances out to cosmological redshifts it is possible to reconstruct the expansion history of the Universe [1, 2, 3]. Over the past decade SNe have helped establish that the expansion of the Universe is accelerating [4, 5, , 6, 7, 8]. Current and proposed SN surveys aim to significantly expand and refine the database of SN measurements, and thereby improve our understanding of the dark energy phenomena thought responsible for the accelerating expansion.

The next generation of $\mathrm{SN}$ observations will attempt to answer a fundamental question about dark energy: whether it is static, indicative of a cosmological constant, or time-evolving, such as quintessence and a wide range of alternative theories [9]. In addition to variations in time, it is also prudent to consider the possibility of spatial variations. Fluctuations of the luminosity as a function of direction on the sky, relative to the global average, may indicate novel physics irrespective of the dark energy equation-ofstate. The aim of this paper is to explore the degree to which future SN data may place interesting limits on the homogeneity and isotropy of dark energy.

Dynamical dark energy is necessarily inhomogeneous; covariance implies that a time-varying field is equivalently spatially-varying. Consequently, fluctuations in dynamical dark energy develop in response to inhomogeneities in the surrounding radiation and matter fields [10,11, 12]. Where there are fluctuations in the dark energy density, there should also be spatial variations in the local expansion rate. These variations may be directly observed as spatial anisotropy of the luminosity distance-redshift relation. In addition, modifications to gravity can masquerade as dark energy (e.g. 13]); spatial fluctuations in the new gravitational fields can leave a similar imprint on the local expansion rate and luminosity distances, and there are ongoing efforts to use fluctuation spectra to test for general departures from General Relativity [14, 15, 16, 17, 18]. Although we do not expect spatial fluctuations in slowly-evolving quintessence fields, or similarly weak modifications of gravity, to have mea- surable impact on the luminosity distance-redshift relation, our lack of understanding of the physics underlying the dark energy suggests that we not preclude observable spatial fluctuations.

Dark energy has also been speculatively tied to the breakdown of two fundamental tenets of cosmology, homogeneity and isotropy. If the observed cosmic acceleration is due to gravitational effects in a strongly inhomogeneous universe [19, 20] then we may expect similar distortions of the expansion rate and luminosity distance. Anisotropic dark energy [21, 22, 23] may be connected to a breakdown of global, statistical isotropy, hints of which have been seen in the CMB 24, 25]. It is conceivable that these features are also present in the expansion rate of the Universe, and will be reflected in residuals in the SN luminosity distance [26]. Furthermore, tests for angular variations in the luminosity distance can be used to constrain novel photon interactions, such as photon-to-axion conversion [27, 28], which can distort our view of the cosmic expansion history.

While we do not yet have an understanding of the physical nature of dark energy, certain candidate models predict spatial fluctuations in the energy density of dark energy, relative to the global average, with amplitude $10^{-4}$ on horizon-size length scales [11, 29]. This scale suggests that the best probe of dark energy clustering may be the $\mathrm{CMB}$, in combination with other measures of large scale structure. An all-sky CMB map can be used to study dark energy anisotropies based on differences in the distance to the last scattering surface in different directions. Unfortunately, the CMB integrates out to $z \sim 1100$, and thus is insensitive to dark energy physics at relatively low redshifts. CMB limits for dark energy horizon-scale rms fractional fluctuations are not expected to be better than $10^{-2}$.

The clustering of dark energy in a supernova sample manifests itself as correlated fluctuations of the observed luminosity residuals. Of course, a variety of conventional gravitational phenomena also distort the paths of photons from distant $\mathrm{SNe}$ [30, 31]. The dominant effect at small angular scales $(\lesssim 5$ arcmin $)$ and high redshift $(z>0.5)$ is the lensing magnification due to the intervening mass fluctuations [32, 33, 34]. This 
lensing effect results in correlated spatial anisotropies in the luminosity distance estimated from nearby $\mathrm{SNe}$ on the sky, and can be measured in terms of magnitude residuals relative to the best-fit cosmological distance relation at a given redshift 35]. Previous studies have shown that a deep SN survey can be used to measure magnification statistics of the Universe [36].

There are also correlated fluctuations due to SachsWolfe and integrated Sachs-Wolfe-like effects, consisting of weighted integrals of time derivatives of the scalar gravitational potential along the line of sight, but these are expected to be too small to be relevant [30]. Finally, there are spatial correlations due to the bulk flows in which the SN host galaxies are embedded [37, 38, 39], although these are only important at lower redshifts $(z<0.2)$. Efforts to discover correlated luminosity fluctuations induced by dark energy must overcome these nuisance effects.

Calculational Method - We calculate the fluctuations in the apparent luminosity due to perturbations in the dark energy component 45]. We also incorporate the effects due to inhomogeneities in the matter, which account for conventional gravitational lensing. We lack a theoretically compelling model for the dark energy, and thus confine ourselves to a purely phenomenological description of dark energy inhomogeneities, motivating a model-independent measurement that can be used to guide future tests of specific models of clustering.

We model the luminosity distance, $d_{L}(z, \hat{\mathbf{n}})$, to a source at redshift $z$ as a function of sky location $\hat{\mathbf{n}}$. In a general Robertson-Walker space-time, the luminosity distance along a given line-of-sight is given by

$$
\begin{aligned}
d_{L}(z, \hat{\mathbf{n}}) & =\frac{(1+z) c}{H_{0} \sqrt{\Omega_{K}}} \sinh \left(\sqrt{\Omega_{K}} \int_{0}^{z} d z^{\prime} /\left[\Omega_{x}\left(z^{\prime}, \hat{\mathbf{n}}\right)\right.\right. \\
& \left.\left.+\Omega_{M}\left(1+z^{\prime}\right)^{3}+\Omega_{K}\left(1+z^{\prime}\right)^{2}\right]^{1 / 2}\right)
\end{aligned}
$$

where $c$ is the speed of light, $H_{0}$ is the Hubble constant today, and $\Omega_{M}, \Omega_{K}$, and $\Omega_{x}$ are the matter, curvature, and dark energy density in units of the critical density. We specialize to the spatially-flat case for this study. We note that for the line-of-sight integral, the relevant densities are the actual densities in the given direction (including the effect of spatial variations), not the globally-averaged densities. The apparent luminosity of a source is given by $L(z, \hat{\mathbf{n}})=$ $\bar{L} \times\left(\bar{d}_{L} / d_{L}(z, \hat{\mathbf{n}})\right)^{2}$ where $\bar{L}$ and $\overline{d_{L}}$ are the mean luminosity and luminosity distance, respectively, defined by the underlying, unperturbed cosmology [33]. Perturbations are given by $\delta_{L}(z, \hat{\mathbf{n}})=[L(z, \hat{\mathbf{n}})-\bar{L}] / \bar{L}$. We build a phenomenological model for the dark energy inhomogeneities, characterizing the fluctuations as $\delta_{x}(z, \hat{\mathbf{n}}) \equiv\left[\Omega_{x}(z, \hat{\mathbf{n}})-\bar{\Omega}_{x}(z)\right] / \bar{\Omega}_{x}(z)$, relative to the mean dark energy density, $\bar{\Omega}_{x}$, at each redshift. We can then write the three dimensional power spectrum for the dark energy fluctuations in Fourier space as $\left\langle\delta_{x}(\mathbf{k}) \delta_{x}\left(\mathbf{k}^{\prime}\right)\right\rangle=(2 \pi)^{2} \delta\left(\mathbf{k}+\mathbf{k}^{\prime}\right) P_{x}(k)$. By observing these perturbations as a function of angular scale and redshift, we can attempt to quantify the underlying inhomogeneity of the dark energy [40].

In the limit of small number statistics, as would be the case with current and next-generation large SN surveys, it may be more practical to measure the angular power spectrum of luminosity fluctuations. Assuming statistical isotropy, the angular power spectrum is given by $\left\langle\left(\delta_{L}\right)_{\ell m}^{\star}\left(\delta_{L}\right)_{\ell^{\prime} m^{\prime}}\right\rangle=C_{\ell}^{L L} \delta_{\ell \ell^{\prime}} \delta_{m m^{\prime}}$, where $\left(\delta_{L}\right)_{\ell m}$ are the multipole moments of the luminosity fluctuations. The expected angular power spectrum of (fractional) luminosity fluctuations, $\delta_{L}$, for a sample of $\mathrm{SNe}$ at redshift $z$, is given by:

$$
C_{\ell}^{L L}=\int d r \frac{W^{2}(r)}{d_{A}^{2}(z)} P_{x}\left(k=\frac{\ell}{d_{A}}, r\right),
$$

where

$$
W(r)=3 \int d r^{\prime} n\left(r^{\prime}\right) \frac{\Omega_{m} H_{0}^{2}}{c^{2} a(r)} \frac{d_{A}(r) d_{A}\left(r^{\prime}-r\right)}{d_{A}\left(r^{\prime}\right)} .
$$

Here $d_{A}(z)$ is the comoving angular diameter distance, $r$ is the comoving radial distance, and $n(r)$ is the radial distribution of SNe.

Equation 2 neglects the contributions of gravitational lensing due to matter inhomogeneities. In what follows we will consider lensing as a source of noise, such that any measurement of $P_{x}(k)$ above this noise represents a measurement of the dark energy inhomogeneity power spectrum. Instead of a specific model [4], we characterize the power spectrum of dark energy fluctuations with a phenomenological model in Fourier space:

$$
\frac{k^{3} P_{x}(k, z)}{2 \pi^{2}}=\delta_{x H}^{2}\left(\frac{c k}{H_{0}}\right)^{n_{x}+3} e^{-k / k_{c}}(1+z)^{-s},
$$

where $\delta_{x H}$ is the amplitude at horizon scales, $n_{x}$ is the tilt to the spectrum, $s$ is the exponent in the growth factor, and $k_{c}$ is the cut-off scale for fluctuations. For quintessence fields the cut-off wavenumber is set by the dynamics of the equation of state. Formally, $P_{x}$ is the power spectrum of an additional clustering component to potential fluctuations, which we evaluate in the conformal-Newtonian/longitudinal gauge (we absorb a prefactor proportional to $\Omega_{x}$ to $\delta_{x H}$ [42]). The parameters $\left(\delta_{x H}, n_{x}, s, k_{c}\right)$ can be constrained with future SN data. Note that while we have written a power spectrum associated with dark energy fluctuations, the same power spectrum may also describe the impact of modifications to gravity, or exotic physics of the dark sector.

Gravitational lensing due to the inhomogeneous matter distribution sets a lower limit on the strength of detectable dark energy fluctuations. The dark energy signal must rise above this amplitude, and thus the $1 \sigma$ upper limit on $\delta_{x H}$ (given other parameters) of a null experiment is given by

$$
\frac{1}{\left(\delta_{x H}^{\min }\right)^{2}}=\sum_{l=l_{\min }}^{l_{\max }}\left(\frac{\partial C_{\ell}^{L L}}{\partial \delta_{x H}}\right)_{\delta_{x H}=0}^{2} \frac{1}{\left(\Delta C_{\ell}^{L L}\right)^{2}},
$$


where

$$
\Delta C_{\ell}^{L L}=\sqrt{\frac{2}{(2 \ell+1) f_{\mathrm{sky}} \Delta \ell}}\left[C_{\ell}^{\mu}+\frac{\sigma_{L}^{2}}{N_{\mathrm{SN}}}\right] .
$$

Here $C_{\ell}^{\mu}$ is the anisotropy power spectrum of SN luminosities expected from cosmic magnification due to mass fluctuations [36], $N_{\mathrm{SN}}$ is the surface density of $\mathrm{SNe}$ (number per steradian), $\sigma_{L}$ is the uncertainty in the luminosity measurement from each $\mathrm{SN}, f_{\text {sky }}$ is the fraction of sky covered by the survey, and $\Delta \ell$ is the binning width in multipole space. When considering the extent to which finite SN surveys can search for inhomogeneities, we take $l_{\min }=180^{\circ} / \theta$ where $\theta$ is the one-sided area of the survey. We assume an uncertainty in the (calibrated) intrinsic luminosity of $\mathrm{SNe}$ given by $\sigma_{L}=0.1$.

Results - Fig. 1 1 summarizes our results, illustrating the sensitivity of future SN surveys to clustering of the dark energy. We show the angular clustering power spectrum of luminosity variations relative to the mean luminosity. The weak lensing power spectrum (thin solid curve) dominates at arcminute angular scales and at high redshifts [36]. We also show examples of dark energy clustering, arising from models with $\delta_{x H}^{2}=10^{-5}, s=2, k_{c}=10^{-1} h \mathrm{Mpc}^{-1}$ for three values of $n_{x}$. These spectra are chosen such that fluctuations in the luminosity residuals become important at large angular scales, and thus dominate over the perturbations due to matter fluctuations (gravitational lensing). For comparison, the amplitude $\delta_{x H}^{2}$ for horizon-scale perturbations, relative to the dark energy density, is of order $\sim 10^{-8}$ for certain dark energy models [11, 29].

For comparison, in Fig. 1 w we also plot the shot-noise for two potential SN surveys (dashed lines): 3,000 SNe in $10 \mathrm{deg}^{2}$, as can be achieved with deep repeated observations from space, and $10^{6} \mathrm{SNe}$ in 20,000 $\mathrm{deg}^{2}$, from a shallow, but wide, SN survey. The latter is equivalent to what can be achieved with the Large Synoptic Survey Telescope (LSST; 43] ). We combine the effects of lensing and shot-noise, generating a total noise estimate in wide multipole bins, following equation (6). The stair-step curves show this binned noise for the two SN samples we consider, where we take $\Delta \ell=2 \ell$ for the bin sizes.

Using the procedure outlined in equations (5) and (6), we have estimated the minimum rms amplitude of dark energy spatial fluctuations at horizon scales that can be detected with future SN samples. A survey of $3,000 \mathrm{SNe}$ in $10 \mathrm{deg}^{2}$ (e.g., from a space-based JDEM 44]) provides limited constraints due to the narrow sky coverage, finding limits of $\delta_{x H}<0.2$ if $n_{x} \sim-3$, and $\delta_{x H}<10^{-4}$ if $n_{x} \sim 0$. A shallow, wide survey of $10^{6} \mathrm{SNe}$ over $20,000 \mathrm{deg}^{2}$ (e.g., as expected from LSST) could potentially limit $\delta_{x H}<3 \times 10^{-3}$ if $n_{x} \sim$ -3 , and $\delta_{x H}<2 \times 10^{-5}$ if $n_{x} \sim 0$. These limits are for a growth exponent of $s=2$ in equation (4). If dark energy fluctuations are redshift-independent

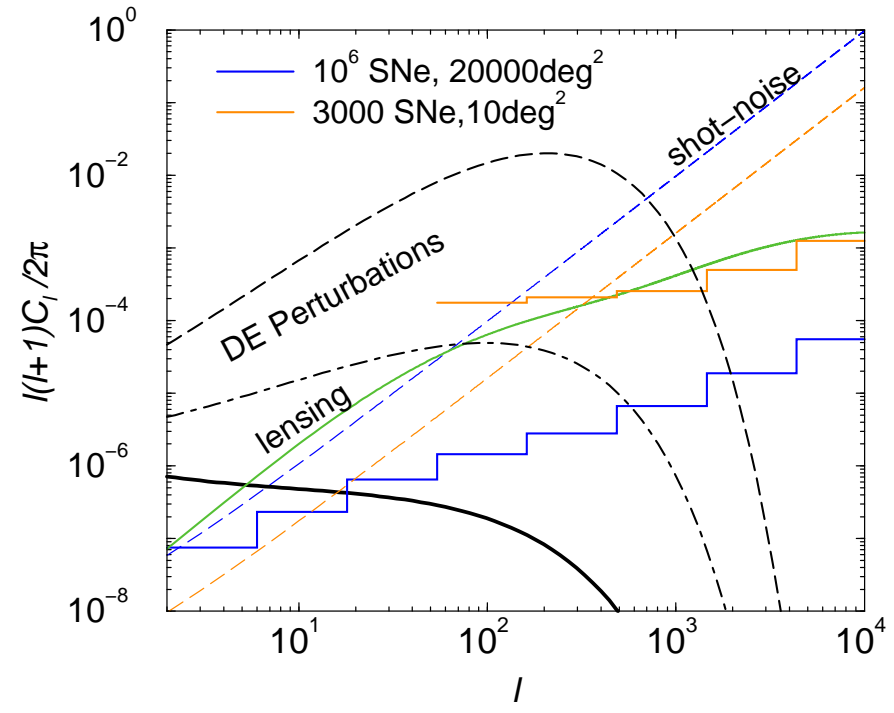

FIG. 1: Angular power spectrum $\left(C_{\ell}^{L L}\right)$ of SNe luminosity, relative to the mean, as a function of redshift. The thin solid "lensing" line is the power spectrum due to gravitational lensing: the luminosity fluctuations generated by magnification associated with foreground mass perturbations. We show three examples of power spectra due to large-scale dark energy inhomogeneities, characterized by (see eq. (4) ) $\delta_{x H}^{2}=10^{-5}, k_{c}=0.1 \mathrm{~h} \mathrm{Mpc}^{-1}, s=2$ and $n_{x}=-2$ (bottom solid line), -1 (middle dot-dashed line), and 0 (top dashed line). The two dashed "shot-noise" curves are for surveys with 3,000 SNe in $10 \mathrm{deg}^{2}$ (bottom) and $10^{6} \mathrm{SNe}$ in $20,000 \mathrm{deg}^{2}$ (top). The two stair-step lines show the total noise in measuring luminosity anisotropies (equation 6), including the contribution from lensing term $C_{l}^{\mu}$. The width of the step is equal to the bin width $\Delta \ell$.

$(s=0)$, then the limits improve by a factor of 2 to 3. Independent of $n_{x}$ and $s$, LSST could limit the dark energy quadrupole $(l=2)$ to a fractional energy density as low as $2 \times 10^{-4}$.

The phenomenological dark energy fluctuation power spectrum may be observationally determined by mapping the luminosity residuals as a function of SN redshift, and then measuring the angular power spectrum of the residuals (i.e., in the same manner that $\mathrm{CMB}$ residuals are treated). The imprint of dark energy perturbations on the $\mathrm{CMB}$, and crosscorrelations with other tracers of large scale structure, is limited by cosmic variance. By using a shallow, allsky SN survey the dark energy perturbation signal is dramatically improved. Thus, beyond the CMB, it is likely that $\mathrm{SN}$ data will become the most powerful probe of anisotropic expansion and dark energy inhomogeneity in the universe.

The multipole moments of the luminosity residuals, $\left(\delta_{L}\right)_{\ell m}$, can be used for cross-correlation studies with other trace fields of the large-scale structure. The moments can be used for additional statistical studies to constrain signatures of global anisotropies, which may be due to anisotropic dark energy or other 
direction-dependent phenomena. In a similar fashion, such studies will benefit from an all-sky SN sample, which could be obtained with a shallow SN survey out to $z \sim 1$ rather than a deep (but narrower) survey that attempts to recover SNe at $z \sim 2$. Alternatively, the absence of SN luminosity fluctuations due to dark energy inhomogeneities consists of a null test of the cosmological constant.

The cosmological community is aggressively developing and perfecting probes of the time dependence of the dark energy equation-of-state. It is to be noted that time-dependent models of the dark energy also necessarily predict spatial dependences. Type Ia supernovae have become one of the most powerful probes of the dark energy equation of state, and are expected to provide precision constraints on possible time variation. We show that these same supernovae also offer one of the most powerful methods to constrain spatially inhomogeneous models of the dark energy.

We have presented a simple phenomenological model for large-scale spatial fluctuations of the dark energy, and have derived limits to how well this model can be constrained with various proposed surveys. It will be useful to quantify the effective variations in dark energy for a variety of theoretical models. In addition, it would be worthwhile to consider other potential cosmological probes of dark energy inhomogeneity, including signatures in weak lensing shear measurements of galaxy shapes, as well as spatial inhomogeneities in baryon acoustic oscillation measurements. The advent of almost all-sky SN samples, such as would be expected from LSST, will sharply constrain the amount of spatial variation in the dark energy.

This work was supported by LANL IGPP Astro1603-07 (DEH \& AC), NSF CAREER AST-0645427 (AC), and AST-0349213 (RRC). AC and DEH thank the Aspen Center for Physics for hospitality while this paper was initiated. AC and RRC thank Caltech for hospitality while this paper was completed.
[1] M. Kowalski et al., arXiv:0804.4142 [astro-ph].

[2] A. G. Riess et al., Astrophys. J. 607, 665 (2004).

[3] J. L. Tonry et al., Astrophys. J. 594, 1 (2003).

[4] A. Riess et al., Astron. J. 116, 1009 (1998)

[5] A. Riess et al., Astron. J. 116, 1009 (1998); S. Perlmutter et al., Astrophys. J. 517, 565 (1999).

[6] W. M. Wood-Vasey et al., arXiv:astro-ph/0701041.

[7] A. G. Riess et al., Astrophys. J. 659, 98 (2007).

[8] P. Astier et al., Astron. Astrophys. 44731 (2006).

[9] D. Sarkar, et al., Phys. Rev. Lett. 100, 241302 (2008).

[10] C. P. Ma, R. R. Caldwell, P. Bode and L. M. Wang, Astrophys. J. 521, L1 (1999).

[11] R. Dave, R. R. Caldwell and P. J. Steinhardt, Phys. Rev. D 66, 023516 (2002).

[12] S. Dutta and I. Maor, Phys. Rev. D 75, 063507 (2007).

[13] J. P. Uzan, Gen. Rel. Grav. 39, 307 (2007).

[14] W. Hu and I. Sawicki, Phys. Rev. D 76, 104043 (2007)

[15] S. F. Daniel, R. R. Caldwell, A. Cooray and A. Melchiorri, Phys. Rev. D 77, 103513 (2008).

[16] B. Jain and P. Zhang, Phys. Rev. D 78, 063503 (2008).

[17] E. Bertschinger and P. Zukin, Phys. Rev. D 78, 024015 (2008).

[18] Ẽ E. E. Flanagan, E. Rosenthal, and I. M. Wasserman, arXiv:0810.0535 [astro-ph]

[19] E. W. Kolb, S. Matarrese and A. Riotto, New J. Phys. 8, 322 (2006).

[20] S. Rasanen, JCAP 0611, 003 (2006).

[21] C. Armendariz-Picon, JCAP 0407, 007 (2004).

[22] R. A. Battye and A. Moss, Phys. Rev. D 74, 041301 (2006).

[23] T. Koivisto and D. F. Mota, JCAP 0806, 018 (2008).

[24] H. K. Eriksen, A. J. Banday, K. M. Gorski, F. K. Hansen and P. B. Lilje, Astrophys. J. 660, L81 (2007).

[25] T. R. Jaffe, A. J. Banday, H. K. Eriksen, K. M. Gorski and F. K. Hansen, Astrophys. J. 629, L1 (2005).

[26] T. Koivisto and D. F. Mota, arXiv:0707.0279 [astro- $\mathrm{ph}$.

[27] C. Csaki, N. Kaloper and J. Terning, Phys. Rev. Lett. 88, 161302 (2002).

[28] Y. S. Song and W. Hu, Phys. Rev. D 73, 023003 (2006).

[29] C. Gordon and D. Wands, Phys. Rev. D 71, 123505 (2005).

[30] C. Bonvin, R. Durrer and M. A. Gasparini, Phys. Rev. D 73, 023523 (2006).

[31] L. Hui and P. B. Greene, Phys. Rev. D 73, 123526 (2006).

[32] J. A. Frieman, arXiv:astro-ph/9608068

[33] D. E. Holz and R. M. Wald, Phys. Rev. D 58, 063501 (1998).

[34] D. Sarkar, A. Amblard, D. E. Holz and A. Cooray, arXiv:0710.4143 [astro-ph].

[35] A. Cooray, D. Huterer and D. Holz, Phys. Rev. Lett. 96, 021301 (2006).

[36] A. Cooray, D. Holz and D. Huterer, Astrophys. J. 637, L77 (2006).

[37] A. Cooray and R. R. Caldwell, Phys. Rev. D 73, 103002 (2006).

[38] J. D. Neill, M. J. Hudson and A. Conley, arXiv:0704.1654 [astro-ph].

[39] C. Gordon, K. Land and A. Slosar, arXiv:0705.1718

[40] M. Blomqvist, E. Mortsell and S. Nobili, arXiv:0806.0496 [astro-ph].

[41] M. Takada, Phys. Rev. D 74, 043505 (2006); W. Hu and R. Scranton, Phys. Rev. D 70, 123002 (2004)

[42] D. F. Mota, D. J. Shaw and J. Silk, Astrophys. J. 675, 29 (2008).

[43] Z. Ivezic, J. A. Tyson, R. Allsman, J. Andrew, R. Angel and f. t. L. Collaboration, arXiv:0805.2366

[44] A. Albrecht et al., arXiv:astro-ph/0609591

[45] It is also possible to perform our analysis in luminosity distance instead of luminosity, with identical results. 